Bull. Austral. Math. Soc.

VOL. 58 (1998) [453-464]

\title{
ANNULAR DEHN FUNCTIONS OF GROUPS
}

\author{
STEPHEN G. Brick AND JoN M. CORSON
}

For a finite presentation of a group, or more generally, a two-complex, we define a function analogous to the Dehn function that we call the annular Dehn function. This function measures the combinatorial area of maps of annuli into the complex as a function of the lengths of the boundary curves. A finitely presented group has solvable conjugacy problem if and only if its annular Dehn function is recursive.

As with standard Dehn functions, the annular Dehn function may change with change of presentation. We prove that the type of function obtained is preserved by change of presentation. Further we obtain upper bounds for the annular Dehn functions of free products and, more generally, amalgamations or HNN extensions over finite subgroups.

\section{INTRODUCTION}

Suppose $G$ is a finitely presented group. It is well-known that the word problem for $G$ is solvable if and only if the Dehn function associated to some and hence any finite presentation is recursive. In this paper we turn to the conjugacy problem. We introduce and study the annular Dehn function, and obtain results analogous to those in $[2]$.

The annular Dehn function is defined in an analogous way to that of the standard Dehn function. Let $K$ be a finite two-complex. Consider a pair of edge-circuits $w$ and $u$ which cobound some singular annulus $A$ in $K$, that is, are freely homotopic in $K$. Define

$$
\Delta_{K}(w, u)=\min \{a(S) \mid S \text { is a singular annulus with boundary } w \cup u\},
$$

where $a(S)$ is the area of the singular annulus, that is, the number of preimage subdisks. Note that the definition could also be given in terms of annular van Kampen diagrams. For convenience sake, we allow the possibility of $w$ or $u$ being the trivial loop. In that case the singular annulus is degenerate, that is, is a singular disk. We define the annular Dehn function of $K$ to be

$$
\begin{aligned}
\gamma_{K}(n)=\max \left\{\Delta_{K}(w, u) \mid w \text { and } u\right. \text { cobound some } \\
\text { singular annulus and }|w|+|u| \leqslant n\} .
\end{aligned}
$$

Received 26th March, 1998

Copyright Clearance Centre, Inc. Serial-fee code: 0004-9729/98 \$A2.00+0.00. 
Since we allow for empty circuits and degenerate annuli, it is clear that $\delta_{K} \leqslant \gamma_{K}$ where $\delta_{K}$ is the ordinary Dehn function.

If $\mathcal{P}$ is a finite presentation then the annular Dehn function can also be defined algebraically. The cobounding edge-circuits are replaced by words representing conjugate group elements and $\Delta_{\mathcal{P}}$ is defined using the minimal number of relators necessary to show two given words represent conjugate elements.

A standard argument (see [7]) shows its conjugacy problem is solvable if and only if the annular Dehn function $\gamma_{\mathcal{P}}$ is recursive. A similar statement is true for finite two-complexes.

An alternate approach to the conjugacy problem is to try to bound the length of the conjugating element and to then make use of the regular Dehn function. Fix a finite generating set $X$ and take the length function $|*|_{X}$ on $G$ determined by it. If $g$ and $h$ are conjugate elements in $G$, let

$$
c(g, h)=\min \left\{|s| \mid g=s h s^{-1}\right\}
$$

and define the annular width function $\sigma_{X}$ to be

$$
\sigma_{X}(n)=\max \{c(g, h)\}
$$

where the maximum is taken over all such elements with $|g|_{X}+|h|_{X} \leqslant n$. As we shall show below, it easy to bound the annular Dehn function in terms of the annular width function and the regular Dehn function. In fact, it works both ways. We shall prove that the annular width function can be bounded by using the annular Dehn function.

Our first result shows that the type of the annular Dehn function does not change with change of presentation. A similar result holds for the annular width function. Here, by "type" we mean the following: We say that $f \preccurlyeq g$ if there are constants $a, b, c$ so that for all $n$ the following holds:

$$
f(n) \leqslant a \cdot n+b \cdot g(c \cdot n)
$$

(here $f, g:\{1,2, \ldots\} \rightarrow \mathbb{N}$ ). We say that $f$ and $g$ are of the same type, and we write $f \equiv g$, if both $f \preccurlyeq g$ and $g \preccurlyeq f$ are true. As is standard practice, when we speak of the annular Dehn function of a group, we are speaking of a function only defined up to equivalence of type.

Standard results about hyperbolic groups show that they have linear annular Dehn functions. And it is a consequence of [6], that quasigeodesic asynchronously combable groups have annular Dehn functions bounded by double exponential functions.

For the regular Dehn function, that is, that associated to disks and the word problem, it is a result of [1] that quasi-isometries preserve the type of function obtained. 
In the case of the annular Dehn function that is not true. In fact, commensurability does not preserve the type of annular Dehn function obtained. This is a consequence of a construction found in [3] and the observation that if $f \equiv g$ and $f$ is bounded by a recursive function then so is $g$.

For our result about amalgamations, we need to recall another notion. We say a function $f$ is subnegative if $f(n)+f(m) \leqslant f(n+m)$, for all $m, n \in \mathbb{N} \backslash\{0\}$. Given a function $h$, we write $\bar{h}$ for the subnegative closure of $h$, that is, the smallest subnegative function greater than or equal to $h$.

We make use of the subnegative closure in the following result: if $G$ is an amalgamation $A *_{C} B$ where $C$ is finite and $A$ and $B$ are finitely presented, then $\gamma_{G} \preccurlyeq \max \left\{\bar{\gamma}_{A}, \bar{\gamma}_{B}\right\}$. There is a similar result for HNN extensions.

It is perhaps worth remarking that our original hope was to obtain a new proof of the solvability of the conjugacy problem for one-relator groups (see [4]). However, we were unable to complete the proof.

In the following, by a cellular map, we mean one transverse to the cell-structure of its target space. In other words, we take the "picture" approach to maps and areas.

\section{INVARIANCE UNDER CHANGE OF PRESENTATION}

Let $K$ and $L$ be finite two-complexes with isomorphic fundamental groups. Fix basepoints. Then there are basepoint-preserving cellular maps $f: K \rightarrow L$ and $g$ : $L \rightarrow K$ so that the induced maps $f_{*}$ and $g_{*}$ of fundamental groups are inverses to one another.

Since $K$ and $L$ are finite complexes, the maps $f$ and $g$ are Lipschitz in the following sense: there is a constant $k$ such that

(i) $|f(w)| \leqslant k \cdot|w|$ for each edge-path $w$ of $K$,

(ii) $|g(w)| \leqslant k \cdot|w|$ for each edge-path $w$ of $L$,

(iii) $a(f(S)) \leqslant k \cdot a(S)$ for each singular surface $S$ in $K$,

(iv) $a(g(S)) \leqslant k \cdot a(S)$ for each singular surface $S$ in $L$.

The Lipschitz constant is the key idea of the proof of the invariance of the annular Dehn function:

THEOREM 1.1. Let $K$ and $L$ be finite connected two-complexes with isomorphic fundamental groups. Then $\gamma_{K} \equiv \gamma_{L}$.

Proof: We start by considering the case where one of the complexes is obtained by collapsing a maximal tree. So assume $T$ is a maximal tree in $K$ and $L=K / T$. Let $\alpha: K \rightarrow L$ be the natural projection map. Since $T$ is a finite tree, there is a bound $c$ to the length of a reduced path in $T$. Let $C=2 c+1$. A loop $w$ of length $\leqslant l$ in $K$ projects to a loop $\alpha(w)$ in $L$ of length $\leqslant l$, while a loop $w$ in $L$ of length $\leqslant l$ may be 
lifted to a loop in $K$ of length $\leqslant C \cdot l$, by lifting the edges one at a time and joining them up by reduced paths in the maximal tree. Abusing notation slightly, we denote the lift by $\alpha^{-1}(w)$. Note that $\alpha^{-1}(w)$ may not project to $w$. However it will project to a loop freely homotopic to $w$, where the homotopy takes place inside the one-skeleton. In other words $w$ may not itself be cyclically reduced. As some of the attaching maps of the two-cells might themselves be not cyclically reduced, we need to allow for this possibility. Also note that we can also project and lift singular annuli without changing their areas.

Suppose $w$ and $u$ are freely homotopic edge-circuits in $L$ with $|w|+|u| \leqslant n$. Then $\alpha^{-1}(w)$ and $\alpha^{-1}(u)$ are freely homotopic edge-circuits in $K$ with $\left|\alpha^{-1}(w)\right|+$ $\left|\alpha^{-1}(u)\right| \leqslant C \cdot n$. Let $S$ be a minimal area singular annulus cobounding $\alpha^{-1}(w)$ and $\alpha^{-1}(u)$. Then $\alpha(S)$ is a singular annulus cobounding $w$ and $u$. Thus it follows that $\gamma_{L}(n) \leqslant \gamma_{K}(C \cdot n)$. Conversely, suppose $w$ and $u$ are freely homotopic edge-circuits in $K$. Then $\alpha(w)$ and $\alpha(u)$ are freely homotopic in $L$. Note that $|\alpha(w)|+|\alpha(u)| \leqslant n$. Let $S$ be a minimal area singular annulus cobounding $\alpha(w)$ and $\alpha(u)$. Lifting $S$ gives a singular annulus of the same area cobounding $w$ and $u$. It follows that $\gamma_{K}(n) \leqslant \gamma_{L}(n)$.

Now we turn to the general case. By the above, we may assume that both $K$ and $L$ have a single vertex. Fix a positive integer $n$. Suppose $w$ and $u$ are freely homotopic edge-circuits in $K$ with $|w|+|u| \leqslant n$. Then $f(w)$ and $f(u)$ are freely homotopic edge-circuits in $L$. Further by the above $|f(w)|+|f(u)| \leqslant k n$. Thus there is a singular annulus $S$ in $L$ cobounding $f(w)$ and $f(u)$ with $a(S) \leqslant \gamma_{L}(k n)$.

Now apply $g$ and we get a singular annulus $g(S)$ cobounding $g(f(w))$ and $g(f(u))$ with $a(g(S)) \leqslant k \gamma_{L}(k n)$. As edge-circuits $g(f(w))$ and $g(f(u))$ are different from $w$ and $u$. But since $g_{*}$ and $f_{*}$ are inverses, $g(f(w))$ and $w$ represent the same element in $\pi_{1}(K)$; a similar statement holds for $g(f(u))$ and $u$ (note: here is where we use the fact that $f$ and $g$ preserve basepoints). So we can find singular disks $D_{1}$ and $D_{2}$ with $D_{1}$ bounding $g(f(w)) \cdot w^{-1}$ and $D_{2}$ bounding $g(f(u)) \cdot u^{-1}$. And since $|w|,|u| \leqslant n$, we may assume $a\left(D_{i}\right) \leqslant \delta_{K}\left(\left(k^{2}+1\right) n\right)$.

Now glue $D_{1}$ and $D_{2}$ onto $g(S)$. This yields a singular annulus cobounding $w$ and $u$. Its area is bounded above by

$$
2 \delta_{K}\left(\left(k^{2}+1\right) n\right)+k \gamma_{L}(k n)
$$

By the invariance of the ordinary Dehn function (see [2] for example), we know $\delta_{K} \equiv \delta_{L}$. And by construction $\delta_{L} \leqslant \gamma_{L}$. Putting this together, after taking a maximum over all such freely homotopic loops, yields $\gamma_{K} \preccurlyeq \gamma_{L}$. By symmetry, $\gamma_{L} \preccurlyeq \gamma_{K}$, and it follows that $\gamma_{K} \equiv \gamma_{L}$.

Let us turn now to invariance of the annular width function. 
THEOREM 1.2. Let $X_{1}$ and $X_{2}$ be two finite generating sets of a group $G$. Then $\sigma_{X_{1}} \equiv \sigma_{X_{2}}$.

Proof: Fix a positive integer $n$. Denote by $|*|_{X_{1}}$ and $|*|_{X_{2}}$ the length functions on $G$ induced by $X_{1}$ and $X_{2}$ respectively. There is a constant $k$ such that

$$
\frac{1}{k} \cdot|g|_{X_{2}} \leqslant|g|_{X_{1}} \leqslant k \cdot|g|_{X_{2}}
$$

for all $g \in G$.

Now assume $g$ and $h$ are conjugate elements of $G$ with $|g|_{X_{1}}+|h|_{X_{1}} \leqslant n$. Then $|g|_{X_{2}}+|h|_{X_{2}} \leqslant k \cdot n$. Choose $s \in G$ with $s g s^{-1}=h$ and $|s|_{X_{2}}=c_{X_{2}}(g, h)$ (that is, $s$ is the shortest word, when measured using $X_{2}$, conjugating $g$ to $h$ ). Then, by minimality,

$$
c_{X_{1}}(g, h) \leqslant|s|_{X_{1}} \leqslant k \cdot|s|_{X_{2}}=k \cdot c_{X_{2}}(g, h) \leqslant k \cdot \sigma_{X_{2}}(k \cdot n)
$$

Taking the maximum over all such $g$ and $h$ yields

$$
\sigma_{X_{1}}(n) \leqslant k \cdot \sigma_{X_{2}}(k \cdot n)
$$

and thus $\sigma_{X_{1}} \preccurlyeq \sigma_{X_{2}}$. Similarly $\sigma_{X_{2}} \preccurlyeq \sigma_{X_{1}}$ and it follows that $\sigma_{X_{1}} \equiv \sigma_{X_{2}}$.

As mentioned in the introduction, the annular width function, together with the regular Dehn function, can be used to bound the annular Dehn function. The idea is simple, $u$ and $w$ represent conjugate elements if and only if there is some $s$ with $W=s w s^{-1} u^{-1}$ representing the identity in the group. Now use the solution of the word problem given by the regular Dehn function, keeping in mind the length of the word $W$.

Conversely, the annular Dehn function yields a bound for the annular width function. Take $m$ to be the maximum length of the relators. Suppose $A$ is a singular annulus. Look at the transverse structure of $A$, that is, its picture. We can compute its width by taking a path connecting the two boundary components of the annulus which is transverse to the arcs of the picture and misses all the subdisks. Now by counting the number of transverse arcs it crosses, we see that each subdisk and the two boundary components may contribute as many points of intersection as half of its length.

It is clear that the above comments yield the following result:

PROPOSITION 1.3. Suppose $\mathcal{P}=\langle X \mid R\rangle$ is a finite presentation and $m$ is the maximum of the lengths of the relators. Then

$$
\begin{aligned}
& \gamma_{\mathcal{P}}(n) \leqslant \delta_{\mathcal{P}}\left(n+2 \sigma_{X}(n)\right) \\
& \sigma_{X}(n) \leqslant \frac{n}{2}+\frac{m}{2} \cdot \gamma_{\mathcal{P}}(n)
\end{aligned}
$$

for all $n$. 


\section{RETRACTS AND DIRECT PRODUCTS}

We turn to the case of a retract of groups:

THEOREM 2.1. Let $H$ be a retract of a finitely presented group $G$. Then $\gamma_{H} \preccurlyeq$ $\gamma_{G}$ and $\sigma_{H} \preccurlyeq \sigma_{G}$.

PRoOF: Let $\rho: G \rightarrow H$ be a retraction of a finitely-presented group $G$ onto a subgroup $H$, with $i: H \subset G$ denoting the inclusion map. Choose a finite presentation for $G$ such that some subpresentation is a presentation of $H$. Then if $(L, K)$ is the pair of associated two-complexes we have $K \subset L$ and there is a cellular retraction $p: L \rightarrow K$ such that $p_{*}=\rho$. Each two-cell $D$ of $L \backslash K$ is mapped by $p$ in a cellular fashion. Let $m$ be the largest area of the maps $p\lceil D: D \rightarrow K$ for all such $D$.

Fix a positive integer $n$. Let $\alpha$ and $\beta$ be freely homotopic edge-circuits in $K$ with $|\alpha|+|\beta| \leqslant n$. Clearly they are also freely homotopic in $L$. Suppose $A$ is an annular diagram in $L$ cobounding $\alpha$ and $\beta$ and of minimal area. Then $p(A)$ is an annular diagram in $K$ cobounding $\alpha$ and $\beta$. Then it is clear that, due to the way $p$ maps the two-cells of $L \backslash K$, we have $a(p(A)) \leqslant m \cdot a(A)$. Thus

$$
\Delta_{K}(\alpha, \beta) \leqslant a(p(A)) \leqslant m \cdot a(A) \leqslant m \cdot \gamma_{L}(n)
$$

And taking the maximum over all such $\alpha$ and $\beta$ yields

$$
\gamma_{K}(n) \leqslant m \cdot \gamma_{L}(n)
$$

whence $\gamma_{K} \preccurlyeq \gamma_{L}$ as desired.

Now to consider the annular width functions, choose finite generating sets $X$ and $Y$ for $G$ and $H$ respectively with $Y \subset X$. Letting $k$ be the maximum of $|\rho(x)|_{Y}$ over all $x \in X$, we see that $|\rho(g)|_{Y} \leqslant k \cdot|g|_{X}$ for all $g \in G$.

Fix a positive integer $n$. Suppose $h_{1}$ and $h_{2}$ are conjugate elements of $H$ with $\left|h_{1}\right|_{Y}+\left|h_{2}\right|_{Y} \leqslant n$. Note that since $Y \subset X$ we also have $\left|h_{1}\right|_{X}+\left|h_{2}\right|_{X} \leqslant n$. Choose $s \in G$ so that $h_{2}=s h_{1} s^{-1}$ and $|s|_{X}=c_{X}\left(h_{1}, h_{2}\right)$ (that is, $s$ is a conjugating element for $h_{1}$ and $h_{2}$ in $G$ of minimal length in terms of $X$ ). Then apply the retraction $\rho$ and we see that $h_{2}=\rho(s) h_{1} \rho(s)^{-1}$. So

$$
c_{Y}\left(h_{1}, h_{2}\right) \leqslant|\rho(s)|_{Y} \leqslant k \cdot|s|_{X}=k \cdot c_{X}\left(h_{1}, h_{2}\right) \leqslant k \cdot \sigma_{X}(n)
$$

and taking the maximum over all such $h_{1}$ and $h_{2}$ yields $\sigma_{Y}(n) \leqslant k \cdot \sigma_{X}(n)$ and hence $\sigma_{Y} \preccurlyeq \sigma_{X}$ as desired.

It turns out that for direct products, the annular width function yields a simpler bound than the annular Dehn function: 
THEOREM 2.2. Suppose $G$ and $H$ are finitely presented groups. Then

$$
\sigma_{G \times H} \equiv \sigma_{G}+\sigma_{H} .
$$

Proof: Since $G \times H$ retracts onto $G$ and also onto $H$, we have by Theorem 2.1 that $\max \left\{\sigma_{G}, \sigma_{H}\right\} \preccurlyeq \sigma_{G \times H}$. But $\sigma_{G}+\sigma_{H} \equiv \max \left\{\sigma_{G}, \sigma_{H}\right\}$, so we get $\sigma_{G}+\sigma_{H} \preccurlyeq \sigma_{G \times H}$.

For the other half of the argument, fix a positive integer $n$, and choose finite generating sets $X$ and $Y$ for $G$ and $H$. Take $Z=X \cup Y$ as the generating set for $G \times H$. Suppose $g_{i} \in G$ and $h_{i} \in H$ for $i=1,2$ with $g_{1} h_{1}$ conjugate to $g_{2} h_{2}$ and with $\left|g_{1} h_{1}\right|_{Z}+\left|g_{2} h_{2}\right|_{Z} \leqslant n$. Then $g_{1}$ is conjugate to $g_{2}$ in $G$ and $h_{1}$ is conjugate to $h_{2}$ in $H$. Note that $\left|g_{1}\right|_{X}+\left|g_{2}\right|_{X} \leqslant n$ and $\left|h_{1}\right|_{Y}+\left|h_{2}\right|_{Y} \leqslant n$. Let $s \in G$ with $g_{2}=s g_{1} s^{-1}$ with $|s|_{X} \leqslant \sigma_{X}(n)$ and $t \in H$ with $h_{2}=t h_{1} t^{-1}$ with $|t|_{Y} \leqslant \sigma_{Y}(n)$. Then $g_{2} h_{2}=s t g_{1} h_{1}(s t)^{-1}$ and

$$
|s t|_{Z}=|s|_{X}+|t|_{Y} \leqslant \sigma_{X}(n)+\sigma_{Y}(n) .
$$

Hence $c_{Z}\left(g_{1} h_{1}, g_{2} h_{2}\right) \leqslant \sigma_{X}(n)+\sigma_{Y}(n)$ and taking maximum over all such $g_{1} h_{1}$ and $g_{2} h_{2}$ yields

$$
\sigma_{Z}(n) \leqslant \sigma_{X}(n)+\sigma_{Y}(n) .
$$

Hence the result follows.

\section{Amalgamations}

We begin with the annular width function of an amalgamation.

THEOREM 3.1. Suppose $G *_{C} H$ is an amalgamated free product where $G$ and $H$ are finitely presented groups and $C$ is a finite group. Then

$$
\sigma_{G *_{C} H} \preccurlyeq \sigma_{G}+\sigma_{H} \equiv \max \left\{\sigma_{G}, \sigma_{H}\right\} .
$$

Proof: Choose finite generating sets $X$ and $Y$ for $G$ and $H$ respectively such that $X \cap Y=C \backslash\{1\}$. Then $Z=X \cup Y$ is a generating set for $G *_{C} H$. We establish the result by showing that $\sigma_{Z} \preccurlyeq \sigma_{X}+\sigma_{Y}$.

Fix a positive integer $n$ and let $g$ and $h$ be conjugate elements of $G{ }^{{ }_{C}} H$ such that $|g|_{Z}+|h|_{Z} \leqslant n$. Write $g=u_{1} u_{2} \cdots u_{p}$ where $u_{1}, \ldots, u_{p}$ is a "reduced sequence," that is, the $u_{i}$ are elements alternately in $G$ and $H$, and if $p>1$, then none of the $u_{i}$ lie in the amalgamated part $C$. We may further assume that $|g|_{Z}=\sum\left|u_{i}\right|$ where $\left|u_{i}\right|$ means either $\left|u_{i}\right|_{X}$ or $\left|u_{i}\right|_{Y}$, depending on whether $u_{i}$ is in $G$ or in $H$. To get such a sequence, write $g$ as a product of generators and their inverses, say $g=x_{1} x_{2} \cdots x_{m}$, where $m=|g|_{Z}$. Then let $u_{1}, u_{2}, \ldots, u_{m}$ be a reduced sequence of "syllables" in $G$ and $H$. 
Assume initially that $g$ and $h$ are cyclically reduced (nontrivial) elements. (Then each cyclic permutation of the sequence $u_{1}, \ldots, u_{p}$ is reduced; see [5, IV.2].) Let $b=\max \left\{c_{Z}\left(a, a^{\prime}\right)\right\}$ where the maximum is taken over the (finite) set of pairs $\left(a, a^{\prime}\right)$ from $C$ such that $a$ and $a^{\prime}$ are conjugate in $G *_{C} H$. Then we claim that

$$
c_{Z}(g, h) \leqslant(b+1) n+2\left[\sigma_{X}(n)+\sigma_{Y}(n)\right] .
$$

Indeed, if $p>1$, then by the conjugacy theorem for amalgamations (see [5, IV.2]), $h$ is obtained by cyclically permuting the product $g=u_{1} \cdots u_{p}$ and conjugating by an element of $C$. That is, $h=(s a)^{-1} g(s a)$ where $s=u_{1} \cdots u_{k}$ (some $k<p$ ) and $a \in C$, and hence, $c_{Z}(g, h) \leqslant|s a|_{Z} \leqslant|g|_{Z}+1 \leqslant n$.

So now suppose $p=1$ and choose $s$ such that $s g s^{-1}=h$. Write $s=s_{1} \cdots s_{q}$, where $s_{1}, \ldots, s_{q}$ is a reduced sequence. Since $h$ is cyclically reduced, if $q=1$, then $g$ and $h$ are conjugate in one of $G$ or $H$, in which case $c_{Z}(g, h) \leqslant \max \left\{\sigma_{X}(n), \sigma_{Y}(n)\right\}$. Otherwise, $q>1$ and $s_{q} g s_{q}^{-1}$ must be contained in $C$, that is, $g$ is conjugate in one of $G$ or $H$ to an element $a \in C$. And similarly $h$ is conjugate in one of $G$ or $H$ to an element $a^{\prime}$ of $C$. Thus, in this case, $c_{Z}(g, h) \leqslant \max \left\{\sigma_{X}(|g|+1), \sigma_{Y}(|g|+1)\right\}+$ $c_{Z}\left(a, a^{\prime}\right)+\max \left\{\sigma_{X}(|h|+1), \sigma_{Y}(|h|+1)\right\} \leqslant b+2 \max \left\{\sigma_{X}(n), \sigma_{Y}(n)\right\}$, completing the claim.

For the general case, note that $g$ and $h$ are conjugate to cyclically reduced elements, say $g_{0}$ and $h_{0}$, and that $c_{Z}\left(g, g_{0}\right) \leqslant|g|_{Z}$ and $c_{Z}\left(h, h_{0}\right) \leqslant|h|_{Z}$. Moreover, $\left|g_{0}\right|_{Z} \leqslant|g|_{Z}$ and $\left|h_{0}\right|_{Z} \leqslant|h|_{Z}$. Thus, by the above, $c_{Z}\left(g_{0}, h_{0}\right) \leqslant(b+1) n+2\left[\sigma_{X}(n)+\sigma_{Y}(n)\right]$. It follows that $c_{Z}(g, h) \leqslant(b+2) n+2\left[\sigma_{X}(n)+\sigma_{Y}(n)\right]$, from which our desired result follows.

We turn now to the annular Dehn function. Our approach is to construct the standard topological model of the amalgamation. This space $X$ contains a two-sided subcomplex $F$ carrying the the amalgamating subgroup. Given a singular annulus over $X$, we use transversality to conclude that the inverse image of $F$ is a collection of arcs and simple closed curves. We then proceed by induction on the number of such arcs and simple closed curves. Note that it is also be possible to argue algebraically as above, using normal forms.

ThEOREM 3.2. Suppose $G *_{C} H$ is an amalgamated free product where $G$ and $H$ are finitely presented groups and $C$ is a finite group. Then

$$
\gamma_{G *_{C} H} \preccurlyeq \bar{\gamma}_{G}+\bar{\gamma}_{H}
$$

ProOF: Suppose $C=\left\{c_{1}, c_{2}, \ldots, c_{d}\right\}$ where $c_{1}$ is the identity. Take finite presentations $\mathcal{P}_{G}=\left\langle x_{i}, c_{j} \mid r_{l}\right\rangle$ and $\mathcal{P}_{H}=\left\langle y_{m}, c_{j} \mid s_{t}\right\rangle$ for $G$ and $H$ respectively, both of whose generating sets contain all the $c_{j}$. Then $\mathcal{P}=\left\langle x_{i}, y_{m}, c_{j} \mid r_{l}, s_{t}\right\rangle$ is a presentation 
for the amalgamation $G *_{C} H$. Let $K_{G}, K_{H}$, and $K$ be the canonical two-complexes of the presentations $\mathcal{P}_{G}, \mathcal{P}_{H}$ and $\mathcal{P}$ respectively. Form a new complex $X$ by joining $K_{G}$ and $K_{H}$ by a bouquet of circles cross an interval, $F \times[-1,1]$, where the circles correspond to the generators $c_{j}$. There is an obvious quotient map that collapses $F \times[-1,1]$ onto $F$, so $K$ is a quotient of $X$.

Fix a positive integer $n$. Given edge circuits $u$ and $v$ in $K$ representing conjugate elements, with $|u|+|v| \leqslant n$, form edge circuits $u^{\prime}$ and $v^{\prime}$ in $X$ representing the same elements. Any singular annulus in $X$ which cobounds $u^{\prime}$ and $v^{\prime}$ can be modified by the quotient map $X \rightarrow K$ to a singular annulus over $K$ cobounding $u$ and $v$. We compose with the quotient map and then collapse the inverse images of the $I$-fibers. Our approach will be to use transversality for singular annuli in $X$ and then look at what happens to the area of the corresponding singular annuli in $K$. In the following we shall omit reference to $X$ and will proceed as if we were using transversality for singular annuli in $K$.

We need first to define a constant $a$. Consider all possible pairs of distinct elements $c_{j}$ and $c_{k}$ of $C$ which are conjugate either in $G$ or in $H$. Take $a$ to be the sum of the areas of minimal cobounding annuli for all such pairs. Since $C$ is finite $a$ is a finite constant.

Given an edge circuit $w$ in $K$, write $w=w_{1} \cdots w_{m}$ where the $w_{i}$ are edge circuits alternately in $K_{G}$ and $K_{H}$. Treating $w$ as a cyclic word, define $L_{w}$ to be the smallest such $m$ (the quantity $L_{w}$ was called the cyclic free product length in [2]). We shall prove that for any such $u$ and $v$,

$$
\Delta_{K}(u, v) \leqslant a+\max \left(\bar{\gamma}_{K_{G}}, \bar{\gamma}_{K_{H}}\right)\left(|u|+|v|+L_{u}+L_{v}\right)
$$

Since $L_{u}+L_{v} \leqslant|u|+|v| \leqslant n$, taking the maximum over all such $u$ and $v$ yields the theorem. For convenience, let $M_{u, v}=a+\max \left(\bar{\gamma}_{K_{G}}, \bar{\gamma}_{K_{H}}\right)\left(|u|+|v|+L_{u}+L_{v}\right)$.

Starting with a singular annulus $A$ cobounding $u$ and $v$, using transversality we may assume that the inverse image of $F$ is a collection of properly embedded arcs and simple closed curves. We can assume that no simple closed curve bounds a disk inside of $A$. We can further assume that each of these arcs and loops is mapped to a single edge in $F$, as each element of $C$ is represented by such an edge. Observe that the number of such arcs is equal to $\left\lfloor L_{u} / 2\right\rfloor+\left\lfloor L_{v} / 2\right\rfloor$ (which is equal to $\left(L_{u}+L_{v}\right) / 2$ provided that $L_{u}, L_{v} \geqslant 2$ ). We establish the desired inequality by using induction on $L_{u}+L_{v}$ to show that the minimal area singular annulus $A$ of this type in $K$ that cobounds $u$ and $v$ has area $\leqslant M_{u, v}$.

If $L_{u}=L_{v}=1$ then there are no arcs in the inverse image of $F$. We have two cases. First suppose that there are no simple closed curves in the inverse image of $F$. Then $u$ and $v$ represent elements of one of the two factors, say for example $G$, and are already conjugate in that factor. Clearly we have $\Delta_{K}(u, v) \leqslant \gamma_{K_{G}}(|u|+|v|) \leqslant M_{u, v}$. 
Now suppose there are some simple closed curves in the inverse image of $F$. Enumerate them as $\alpha_{1} \ldots, \alpha_{k}$. Assume they are indexed so that $\alpha_{t}$ is next to $\alpha_{t+1}$, that is, the subregion bounded by $\alpha_{t}$ and $\alpha_{t+1}$ misses the other $\alpha_{i}$ 's. Also assume that $u$ is next to $\alpha_{1}$ and $\alpha_{k}$ is next to $v$. We shall bound the area of $A$ by splitting it up into three regions, the region $A_{1}$ bounded by $u$ and $\alpha_{1}$, the region $A_{2}$ bounded by $\alpha_{1}$ and $\alpha_{k}$, and the region $A_{3}$ bounded by $\alpha_{k}$ and $v$.

Each $\alpha_{i}$ maps to an edge in $F$ representing an element of $C$. We can assume that no element of $C$ is mapped to more than once. For if $\alpha_{i}$ and $\alpha_{j}$ both map to the same element of $C$, then we can cut out the annular region between $\alpha_{i}$ and $\alpha_{j}$, glue $\alpha_{i}$ and $\alpha_{j}$ together, and obtain a singular annulus for $u$ and $v$ with fewer simple closed curve components. It is then easy to see that the area of $A_{2}$, the region bounded by $\alpha_{1}$ and $\alpha_{k}$, is less than or equal to our constant $a$, by construction.

As for the areas of regions $A_{1}$ and $A_{3}$, merely observe that they map entirely into either $K_{H}$ or $K_{G}$ and their boundaries map to elements having lengths bounded by $|u|+1$ and $|v|+1$ respectively. Thus the area of $A_{1}$ is bounded above by $\max \left(\bar{\gamma}_{K_{G}}, \bar{\gamma}_{K_{H}}\right)(|u|+1)$ and that of $A_{2}$ by $\max \left(\bar{\gamma}_{K_{G}}, \bar{\gamma}_{K_{H}}\right)(|v|+1)$. Using subnegativity, noting that $L_{u}+L_{v}=2$, and including our bound for the area of $A_{2}$ yields the desired result that the area of $A$ is bounded above by $M_{u, v}$.

Now assume $L_{u}+L_{v}>2$. If there are any arcs connecting one of the boundary components to itself, say that mapping to $u$, take an innermost one, $p$. The arc, $p$, together with a portion of the boundary of the annulus, say $w$, bounds a disk $D$ that contains no other arcs or simple closed curves. The disk $D$ maps entirely into either $K_{H}$ or $K_{G}$. Say it maps into $K_{G}$. Note that the boundary of $D$ is mapped to a word of length $|w|+1$ and its area is thus bounded by $\delta_{K_{G}}(|w|+1 \mid) \leqslant \gamma_{K_{G}}(|w|+1 \mid)$. We need only bound the area of $A \backslash$ Int $D$, which is a subannulus $A^{\prime}$ cobounding edge-circuits $u_{1}$ and $v$ with $L_{u_{1}}+L_{v} \leqslant L_{u}+L_{v}-1$ (if $L_{u}=2$ then $L_{u_{1}}=1$, otherwise $L_{u_{1}}=L_{u}-2$ ). By induction, the area of $A^{\prime}$ in $K$ is bounded above by

$$
M_{u_{1}, v}=a+\max \left(\bar{\gamma}_{K_{G}}, \bar{\gamma}_{K_{H}}\right)\left(\left|u_{1}\right|+|v|+L_{u_{1}}+L_{v}\right)
$$

The area of $A$, being the union of $A^{\prime}$ and $D$, is therefore bounded above by

$$
a+\max \left(\bar{\gamma}_{K_{G}}, \bar{\gamma}_{K_{H}}\right)\left(\left|u_{1}\right|+|v|+L_{u_{1}}+L_{v}\right)+\gamma_{K_{G}}(|w|+1 \mid)
$$

Now use subnegativity and the facts that $\left|u_{1}\right|+|w|=|u|$ and $L_{u_{1}}+1 \leqslant L_{u}$ to obtain the desired result.

On the other hand, suppose there are arcs in the inverse-image of $F$ but no arc connects a boundary component to itself. By cutting along each arc we obtain a collection $D_{1}, \ldots, D_{k}$ of subdisks, where $k=L_{u}=L_{v}$. The components 
of $D_{i} \cap \partial A$ are mapped to subpaths $u_{i}$ and $v_{i}$ of $u$ and $v$ respectively. Since $D_{i}$ is mapped entirely into one of $K_{G}$ or $K_{H}$, it follows that the area of $D_{i}$ is $\leqslant \max \left(\bar{\delta}_{K_{G}}, \bar{\delta}_{K_{H}}\right)\left(\left|u_{i}\right|+\left|v_{i}\right|+2\right) \leqslant \max \left(\bar{\gamma}_{K_{G}}, \bar{\gamma}_{K_{H}}\right)\left(\left|u_{i}\right|+\left|v_{i}\right|+2\right)$. By subnegativity and the facts that $|u|=\left|u_{1}\right|+\cdots+\left|u_{k}\right|$ and $|v|=\left|v_{1}\right|+\cdots+\left|v_{k}\right|$, it follows that the area of $A$ is bounded above by $\max \left(\bar{\delta}_{K_{G}}, \bar{\delta}_{K_{H}}\right)(|u|+|v|+2 k)$. Since $2 k=L_{u}+L_{v}$, our result follows.

In the case of a free product, that is, where $C$ is trivial, we can use Theorem 2.1 and say a bit more:

Corollary 3.3. Suppose $G$ and $H$ are finitely presented groups. Then

$$
\sigma_{G * H} \equiv \sigma_{G}+\sigma_{H} \quad \text { and } \quad \gamma_{G}+\gamma_{H} \preccurlyeq \gamma_{G * H} \preccurlyeq \bar{\gamma}_{G}+\bar{\gamma}_{H}
$$

For HNN extensions, we have the following result. The proof is similar to that of Theorems 3.1 and 3.2, with free product length replaced by HNN-length. We omit the details.

Theorem 3.4. Suppose $G *_{C} \phi$ is an HNN extension where $G$ is a finitely presented group and $C$ is a finite group. Then

$$
\sigma_{G *_{C} \phi} \preccurlyeq \sigma_{G} \text { and } \gamma_{G *_{C} \phi} \preccurlyeq \bar{\gamma}_{G} .
$$

Using induction, we can also obtain a result for the fundamental group of a finite graph of groups where the edge groups are all finite. We leave the details of the statement and proof to the reader.

\section{EXAMPles}

We conclude with a few examples illustrating our results and mention some subsequent work that we are pursuing. Throughout this section, as elsewhere, all groups are assumed to be finitely presented.

As noted in the introduction, hyperbolic groups are those groups whose Dehn functions are linear, and they are also precisely the groups whose annular Dehn functions are linear. In particular, for a hyperbolic group, the Dehn function is इ-equivalent to the annular Dehn function. It seems interesting to ask what other groups have this property. A sufficient condition for this property is that the annular width function be linear (see Proposition 1.3). That is, if $G$ is a group such that $\sigma_{G}(n) \equiv n$, then $\delta_{G} \equiv \gamma_{G}$.

Regarding the class of groups that have linear annular width functions, first of all note that it clearly contains all (finitely generated) Abelian groups and it includes all hyperbolic groups by the general fact that $\sigma_{G} \preccurlyeq \gamma_{G}$; see Proposition 1.3. Furthermore, 
the property of having a linear annular width function is preserved under forming direct products (by Theorem 2.2) and free products (by Corollary 3.3) and taking retracts (by Theorem 2.1). And the fundamental group of a finite graph of such groups with finite edge groups also has linear annular width function (by Theorems 3.1 and 3.4).

In particular, a direct product of finitely many hyperbolic groups has a linear annular width function. Hence, for any such group $G, \gamma_{G}(n) \equiv \delta_{G}(n) \preccurlyeq n^{2}$ (by [2, Proposition 2.1]). Moreover, if $G$ contains a $\mathbb{Z} \times \mathbb{Z}$ subgroup, then $G$ is not hyperbolic and hence $\delta_{G}(n) \equiv n^{2}$ (as a subquadratic Dehn function is known to imply hyperbolicity). Thus $\gamma_{G}(n) \equiv n^{2}$, unless $G$ is virtually cyclic (and hence hyperbolic).

It is easy to show, by an argument similar to the proof of Theorem 3.1 , that if $G *_{C} H$ is an amalgamation such that $C$ lies in the centre of $G$ and in the centre of $H$, then $\sigma_{G *_{C} H} \preccurlyeq \sigma_{G}+\sigma_{H}$. In particular, every amalgamated free product of Abelian groups has a linear annular width function. The situation for HNN extensions of Abelian groups is more complicated. As a test case, we consider, in a separate paper, group extensions of free Abelian groups by $\mathbb{Z}$. It turns out that for such an extension $G$, the annular Dehn function is always bounded above by an exponential function, and is bounded by a polynomial function if $G$ is nilpotent.

\section{REFERENCES}

[1] J.M. Alonso, 'Inegalités isopérimétriques et quasi-isométries', C.R. Acad. Sci. Paris 311 (1990), 761-764.

[2] S.G. Brick, 'On Dehn functions and products of groups', Trans. Amer. Math. Soc. 335 (1993), 369-384.

[3] D. Collins and C.F. Miller, III, 'The conjugacy problem and subgroups of finite index', Proc. London Math. Soc. 34 (1977), 535-556.

[4] A. Juhàsz, 'Solution of the conjugacy problem in one-relator groups', in Proceedings of the Workshop on Algorithmic Problems, Math. Sci. Res. Inst. Publ. 23 (Springer-Verlag, Berlin, Heidelberg, New York, 1991).

[5] R.C. Lyndon and P.E. Schupp, Combinatorial group theory (Springer-Verlag, Berlin, Heidelberg, New York, 1977).

[6] W.D. Neumann, 'Asynchronous combings of groups', Intnat. J. Algebra Comput. 2 (1992), 179-185.

[7] S.J. Pride, 'Star-complexes, and the dependence problems for hyperbolic complexes', Glasgow Math. J. 30 (1988), 155-170.

\author{
Department of Mathematics and Statistics \\ University of South Alabama \\ Mobile, AL 36688 \\ United States of America \\ e-mail: brick@mathstat.usouthal.edu
}

\author{
Department of Mathematics \\ University of Alabama \\ Tuscaloosa AL 35487-0350 \\ United States of America \\ e-mail: jcorson@mathdept.as.ua.edu
}

